\section{Retinal tears after posterior vitreous detachment and vitreous hemorrhage in patients on systemic anticoagulants}

Cincinnati Eye Institute and University of Cincinnati Department of

Ophthalmology, Cincinnati, $\mathrm{OH}$, USA

Correspondence:

CD Riemann, Cincinnati Eye Institute, 1945 CEI Drive, Cincinnati, OH 45242, USA Tel: + 1513984 5133;

Fax: + 15139844494

E-mail: criemann@

cincinnatieye.com

Received: 4 November 2010 Accepted in revised form: 16 March 2011

Published online: 13 May 2011

Meeting Presentation: This paper was partially presented as a poster presentation to the AAO, November 2007 meeting. Copyright release from Ophthalmology Journal received.

\begin{abstract}
Aims or Purpose To determine the rate of retinal tears (RTs) after posterior vitreous detachment (PVD) and vitreous hemorrhage (VH) in patients on systemic anticoagulants. Methods In all, 260 eyes of 260 patients with an acute PVD and VH were followed for evidence of an RT or detachment. Patients were divided into those taking systemic anticoagulants and those not taking anticoagulants.

Results A total of 137 patients (53\%) were taking anticoagulants, 123 (47\%) were not. Overall, $72 \%$ of patients not taking any anticoagulant had evidence of an RT, whereas $46 \%$ of patients taking an anticoagulant had an RT ( $P$-value 0.0002$)$. Also, $37 \%$ of patients not taking an anticoagulant had a retinal detachment (RD), whereas $23 \%$ of patients taking any anticoagulant had an RD ( $P$-value 0.01$)$. Conclusions In patients with an acute PVD and VH using anticoagulants, RTs and RDs were common. Anticoagulation status may be an important contributing factor in predicting the incidence of an RT or detachment.

Eye (2011) 25, 1016-1019; doi:10.1038/eye.2011.106; published online 13 May 2011
\end{abstract}

Keywords: retinal tears; vitreous hemorrhage; posterior vitreous detachment

\section{Introduction}

Symptomatic acute posterior vitreous detachment (PVD) is a common diagnosis in the ophthalmology clinic. ${ }^{1}$ The natural history of this normal age-related consequence of vitreous liquefaction can be complicated by tractional retinal breaks and subsequent retinal
AA El-Sanhouri, RE Foster, MR Petersen, RK Hutchins, DM Miller, TM Evans, $\mathrm{N}$ Trichopoulos and CD Riemann detachment (RD). ${ }^{2-6}$ The key task for the clinician faced with such a patient is to determine the presence or absence of such breaks and treat, or refer for treatment, appropriately. Vitreous hemorrhage $(\mathrm{VH})$ in the context of acute PVD is a known clinically significant risk factor for the presence of peripheral retinal breaks. ${ }^{7}$ Patients with an acute PVD and VH are followed more closely than those without because of this higher rate of retinal tears (RTs).

The regular use of aspirin (ASA), coumadin, and clopidogrel has increased over the past several decades because of the increasingly recognized benefits of systemic anticoagulation in the management of medical disease, including cardiac and cerebrovascular disease. The associated increased risk of bleeding complications is also well known. Non-steroidal anti-inflammatory drugs (NSAIDs) also have antiplatelet properties, and their use has increased in the treatment of arthritis. ${ }^{8-19}$

In this study, we examine the relationship between systemic anticoagulant and antiplatelet usage and the prevalence of retinal breaks and detachment in patients with $\mathrm{VH}$ in the setting of an acute PVD.

\section{Materials and methods}

This was a consecutive observational case series. All patients signed an informed consent to be treated and all data were collected and analyzed in compliance with the Health Insurance Portability and Accountability Act (HIPAA). All patients evaluated by one of six vitreoretinal surgeons in university and private practice settings from August 1999 to January 2006 were considered for entry in the study. 
Inclusion criteria were as follows: an acute symptomatic PVD, a VH easily visible by indirect ophthalmoscopy with a 20-diopter lens, and the ability to give an accurate history and tolerate repeated examinations of the peripheral retina. Exclusion criteria were as follows: a history of recent trauma, concurrent retinal vascular disease, retinopathy, uveitis, any condition precluding a detailed examination of the peripheral retina, and an inability to return for follow-up.

The patients age, sex, lens status, eye laterality, anticoagulation use, grade of $\mathrm{VH}$, and the presence and geometry of peripheral retinal pathology were tabulated. A retinal tear (RT) was defined as a horseshoe flap tear or operculated full thickness RT with or without associated RD. Atrophic holes associated with lattice degeneration were presumed to have predated the PVD and were not counted as an RT. If subretinal fluid was present but was less than 2-disc diameter (DD), the patient was counted as having an RT without RD. If the subretinal fluid was between 2 and $5 \mathrm{DD}$, the patient was counted as having RD and further counted as having a subclinical RD (sRD). If more than 5 DD of subretinal fluid was present, the patient was counted as having RD and also as having a clinical RD (cRD). By definition, therefore, patients with RT included all patients with retinal breaks alone, patients with sRD, and patients with cRD. Patients with RD included all patients with sRD and cRD.

Fellowship-trained retinal surgeons serially examined all patients until the peripheral retinal status could be absolutely determined to either have or not have retinal breaks. All RTs and detachments were treated with laser, cyrotherapy, or surgery as clinically appropriate. Surgeries performed included pneumatic retinopexy, scleral buckling, scleral buckling with vitrectomy, and pars plana vitrectomy alone. The severity of $\mathrm{VH}$ was subjectively graded at the presenting visit from trace to $4+$ by the examining surgeon.

The type of anticoagulation and/or antiplatelet drug that the patient was using was recorded and included the following: coumadin, ASA, NSAIDs, clopidogrel, and pentoxyphylline. For the purpose of this study, anticoagulation therapy and antiplatelet therapy are grouped together and hereafter referred to as anticoagulation (AC) therapy.

All data were tabulated in Microsoft excel, and all statistical analysis was accomplished using $\chi^{2}$ and ANOVA analyses where appropriate. SPSS version 8.0 for Windows (SPSS Inc., Chicago, IL, USA) was used for statistical analysis.

\section{Results}

A total of 260 eyes of 260 patients were enrolled. There were 127 (49\%) women and 133 (51\%) men. Patient age ranged from 34 to 100 years (mean 62, median 60). In all, $114(44 \%)$ eyes were right eyes and $146(56 \%)$ eyes were left eyes. A total of 211 (81.2\%) eyes were phakic, 48 eyes $(18.5 \%)$ had posterior chamber intraocular lenses, and one eye $(0.4 \%)$ had an anterior chamber lens. Follow-up ranged from 0 to 1077 days (mean 85, median 33). All patients were followed until the $\mathrm{VH}$ had cleared and the anatomical status of the peripheral retina could be absolutely determined. For patients with obvious pathology, this was on the day of presentation (therefore 0 days of follow-up). No patients were lost to follow-up.

AC use was common in our cohort. Of the 260 enrolled patients, $123(47 \%)$ were taking AC medication and 137 $(53 \%)$ were not.

RTs, sRD, and cRD were also common in our cohort. Of the 260 total patients, $156(60 \%)$ had RT and $104(40 \%)$ did not. In all, 77 patients (30\%) had RT without RD, 79 (30\%) had RD, 55 (21\%) had sRD, and 24 (9\%) had cRD. All RTs and sRDs were successfully treated with laser or cryoretinopexy, and all cRD were successfully operated with one surgery.

The prevalence of RT was notably lower in patients taking AC therapy compared with patients not taking AC agents. Of the 123 patients taking AC, 57 (46\%) had an RT and 66 (54\%) did not. Of the 137 patients not taking AC therapy, 99 (72\%) had an RT and 38 (28\%) did not. (Relative risk $(\mathrm{RR})=0.64 ; P=0.00002)$.

The prevalence of RD (sRD or cRD) was notably lower in patients taking $\mathrm{AC}$ therapy compared with patients not taking $\mathrm{AC}$ agents. Of the 123 patients taking $\mathrm{AC}, 28$ (23\%) had an RD and $95(77 \%)$ did not. Of the 137 patients not taking AC therapy, $51(37 \%)$ had an RD and $86(63 \%)$ did not. $(\mathrm{RR}=0.62 ; P=0.01)$.

The prevalence of $\mathrm{cRD}$ was lower in patients taking AC therapy compared with patients not taking AC agents; however, this difference was not statistically significant. Of the 123 patients taking AC, $8(7 \%)$ had a cRD and 115 (93\%) did not. Of the 137 patients not taking AC therapy, $16(12 \%)$ had a cRD and $121(88 \%)$ did not. $(\mathrm{RR}=0.58 ; P=0.08)$.

Tabulation of the incidences of RT, RD, and $\mathrm{cRD}$ as a function of specific AC drug taken is shown in the table. The prevalence of RD was lower in all groups but this trend only reached statistical significance for the NSAID patients ( $R R=0.43, P=0.01, n=45)$. The prevalence of cRD was similarly lower in all groups but did not reach statistical significance for any subgroup analyzed (Table 1).

\section{Discussion}

Our study examines variations in the prevalence of peripheral retinal pathology in patients with acute PVD and $\mathrm{VH}$ as a function of differing systemic AC regimens. 
Table 1 Summary of results

\begin{tabular}{|c|c|c|c|c|c|c|c|c|}
\hline & \multirow{2}{*}{$\begin{array}{c}\text { Total } \\
\mathrm{N}\end{array}$} & \multirow{2}{*}{$\begin{array}{l}\text { None } \\
\text { N (\%) }\end{array}$} & \multicolumn{2}{|c|}{$R T$} & \multicolumn{2}{|c|}{ Any $R D$} & \multicolumn{2}{|c|}{ Clinical RD } \\
\hline & & & $\mathrm{N}(\%)$ & $\begin{array}{c}\text { P-value } \\
\text { (vs no } A C \text { ) }\end{array}$ & $\mathrm{N}(\%)$ & $\begin{array}{c}\text { P-value } \\
\text { (vs no } A C \text { ) }\end{array}$ & $\mathrm{N}(\%)$ & $\begin{array}{c}\text { P-value } \\
\text { (vs no } A C \text { ) }\end{array}$ \\
\hline Vitreous hemorrhage & 260 & $104(40)$ & $156(60)$ & & $79(30)$ & & $24(9)$ & \\
\hline No anticoagulant (AC) therapy & 137 & $38(28)$ & $99(72)$ & & $51(37)$ & & $16(12)$ & \\
\hline Any anticoagulant therapy & 123 & $66(54)$ & $57(46)$ & 0.00002 & $28(23)$ & 0.01 & $8(7)$ & 0.08 \\
\hline ASA & 77 & $45(58)$ & $32(42)$ & 0.00001 & $19(25)$ & 0.06 & $6(8)$ & 0.24 \\
\hline Other NSAID & 45 & $21(47)$ & $24(53)$ & 0.019 & $7(16)$ & 0.01 & $2(4)$ & 0.09 \\
\hline Coumadin & 11 & $8(73)$ & $3(27)$ & 0.0019 & $3(27)$ & 0.51 & $1(9)$ & 0.71 \\
\hline
\end{tabular}

Our data revealed a clinically relevant decreased incidence of RT and RD, and suggested a decreased incidence of $\mathrm{cRD}$ if patients were anticoagulated. The rate of RT and RD was substantially elevated enough to warrant meticulous evaluation of the peripheral retina in all patients irrespective of whether they were taking AC.

The overall frequency of retinal breaks in this observational case series $(60 \%)$ is consistent with previous reports. $^{2-7}$

This study does not provide any evidence of causation of the observed effect. It is unclear whether the AC medications themselves, the medical conditions for which they are being taken, or perhaps another completely unrelated confounding factor is the underlying reason for the observed differences. The anticoagulant effects of coumadin, the antiplatelet effects of ASA, and the antiplatelet side effects of other NSAIDs are well established. ${ }^{17-19}$ We believe that the lower risk of $\mathrm{RT}$ and RD in patients taking AC is not reflective of a protective effect against these peripheral retinal pathologies by AC and speculate that the microtraumas associated with separation of the posterior hyaloid from the retina may result in a clinically evident $\mathrm{VH}$ more often in AC patients.

Our data seem to suggest that the more potently anticoagulating an agent is (coumadin $>$ ASA $>$ NSAIDs) ${ }^{20}$ the more pronounced the clinical effect in the reduction of $\mathrm{RT}$ prevalence $(\mathrm{RR}=0.37,0.58,0.73$ respectively). The presence or absence of other known risk factors for RT and RD (refraction, lattice, contralateral $\mathrm{RD}$, family history) were non-uniformly captured in the charts and were therefore not analyzed or reported. These limitations of our study are certainly typical for retrospective case series.

The incidence of retinal breaks from PVD has been estimated at $2-4 \%$ when $\mathrm{VH}$ is not present and $70 \%$ when there is an associated $\mathrm{VH}^{8-10}$ The frequency of retinal breaks in our patients who were taking AC (46\%) was significantly lower than the frequency in our patients not taking AC (72\%). However, this is still markedly higher than the $2-4 \%$ frequency in patients without $\mathrm{VH}$. The presence of $\mathrm{VH}$ in the setting of PVD in patients taking anticoagulant medications thus continues to signify a markedly higher risk of associated retinal breaks (approximately 12-fold). Although our data clearly suggest a significant non-uniform incidence of retinal breaks among patients with PVD and VH taking AC medications, this study lacks the prospective design and sufficient numbers to make statistically meaningful inferences regarding the comparative effects of individual agents.

The use of anticoagulants and medications with anticoagulant side effects is common in patients with PVD and VH, as is serious sight-threatening peripheral retinal pathology. The lower frequency of such pathology in our series of patients with PVD and VH concurrently taking AC in no way obviates the need for meticulous, complete, and serial examinations of the retinal periphery in all these patients until the peripheral retinal anatomy can be absolutely confirmed. VH is still a strong indicator of RT or detachment, and anticoagulation does not reduce that rate sufficiently to attribute a PVD-associated VH to anticoagulation without excluding a tear.

\section{Summary}

What was known before

- Vitreous hemorrhage (VH) in the context of acute posterior vitreous detachment (PVD) is a known clinically significant risk factor for the presence of peripheral retinal breaks.

What this study adds

- Patients with a VH and an acute PVD have a lower incidence of retinal tears if taking systemic anticoagulants than those not taking such medications.

\section{Conflict of interest}

The authors declare no conflict of interest. 


\section{References}

1 Mirshahi A, Hoehn F, Lorenz K, Hattenbach LO. Incidence of posterior vitreous detachment after cataract surgery. J Cataract Refract Surg 2009; 35: 987-991.

2 Boldrey EE. Risk of retinal tears in patients with vitreous floaters. Am J Ophthalmol 1983; 96: 783-787.

3 Brod RD, Lightman DA, Packer AJ, Saras HP. Correlation between vitreous pigment granules and retinal breaks in eyes with acute posterior vitreous detachment. Ophthalmology 1991; 98: 1366-1369.

4 Tasman WS. Posterior vitreous detachment and peripheral retinal breaks. Trans Am Acad Ophthalmol Otolaryngol 1968; 72: 217-224.

5 Dayan MR, Jayamanne DG, Andrews RM, Griffiths PG. Flashes and floaters as predictors of vitreoretinal pathology: is follow-up necessary for posterior vitreous detachment? Eye 1996; 10: 456-458.

6 Byer NE. Natural history of posterior vitreous detachment with early management as the premier line of defense against retinal detachment. Ophthalmology 1994; 101: 1503-1514.

7 Sarrafizadeh R, Hassan TS, Ruby AJ. Incidence of retinal detachment and visual outcome in eyes presenting with posterior vitreous separation and dense fundusobscuring vitreous hemorrhage. Ophthalmology 2001; 108: 2273-2278

8 Goodman SG, Huang W, Yan AT, Budaj A, Kennelly BM, Gore JM et al. The expanded Global Registry of Acute Coronary Events: baseline characteristics, management practices, and hospital outcomes of patients with acute coronary syndromes. Am Heart J 2009; 158: 193-201.

9 Mauskopf JA, Boye KS, Schmitt C, McCollam P, Birt J, Juniper MD et al. Adherence to guidelines for sensitivity analysis: cost-effectiveness analyses of dual oral antiplatelet therapy. J Med Econ 2009; 12: 141-153.
10 Ross JS, Halm EA, Bravata DM. Use of stroke secondary prevention services: are there disparities in care? Stroke 2009; 40: 1811-1819.

11 Firestone B, Mold JW. Type 2 diabetes: which interventions best reduce absolute risks of adverse events? J Fam Pract 2009; 58: E1.

12 Satiani B. A Medicare primer. J Vasc Surg 2009; 50: 453-460.

13 Di Minno MN, Prisco D, Ruocco AL, Mastronardi P,

Massa S, Di Minno G. Perioperative handling of patients on antiplatelet therapy with need for surgery. Intern Emerg Med 2009; 4: 279-288.

14 White RL, Cohen SP. Return-to-duty rates among coalition forces treated in a forward-deployed pain treatment center: a prospective observational study. Anesthesiology 2007; 107: 872-874.

15 Charles S, Rosenfeld PJ, Gayer S. Medical consequences of stopping anticoagulant therapy before intraocular surgery or intravitreal injections. Retina 2007; 27(7): 813-815.

16 Benzimra JD, Johnston RL, Jaycock P, Galloway PH, Lambert G, Chung AK et al. The Cataract National Dataset electronic multicentre audit of 55,567 operations: antiplatelet and anticoagulant medications. Eye 2009; 23: 10-16.

17 Beyan E, Beyan C, Vaizoglu SA. Predisposing preventable factors in patients with bleeding due to warfarin usage: evaluation of 114 patients. Clin Appl Thromb Hemost 2009; 16(6): 684-687.

18 Yoo SH, Nah HW, Jo MW, Kang DW, Kim JS, Koh JY et al. Age and body weight adjusted warfarin initiation program for ischaemic stroke patients. Eur J Neurol 2009; 16(10): 1100-1105.

19 Lind M, Boman K, Johansson L, Nilsson TK, Ohlin AK, Birgander LS et al. Thrombomodulin as a marker for bleeding complications during warfarin treatment. Arch Intern Med 2009; 169: 1210-1215.

20 Segal JB, McNamara RL, Miller MR, Kim N, Goodman SN, Powe NR et al. Prevention of thromboembolism in atrial fibrillation. A meta-analysis of trials of anticoagulants and antiplatelet drugs. J Gen Intern Med 2000; 15: 56-67. 